\title{
Water-compatible temperature and magnetic dual-responsive molecularly imprinted polymers for recognition and extraction of bisphenol A
}

\author{
Xiaqing Wu $\mathbf{W}^{\mathrm{a}, \mathrm{b}}$, Xiaoyan Wang ${ }^{\mathrm{a}}$, Wenhui $\mathrm{Lu}^{\mathrm{a}}$, Xinran Wang ${ }^{\mathrm{a}, \mathrm{b}}$, Jinhua $\mathrm{Li}^{\mathrm{a}}$, Huiyan You ${ }^{\mathrm{b}, * *}$, \\ Hua Xiong ${ }^{\mathrm{c}}$, Lingxin Chen ${ }^{\mathrm{a}, \mathrm{d}, *}$ \\ ${ }^{a}$ Key Laboratory of Coastal Environmental Processes and Ecological Remediation, Yantai Institute of Coastal Zone Research, Chinese Academy of Sciences, \\ Yantai 264003, China \\ ${ }^{\mathrm{b}}$ Environmental and Chemical Engineering College, Dalian University, Dalian 116622, China \\ ' State Key Laboratory of Food Science and Technology, Nanchang University, Nanchang 330047, China \\ d College of Chemistry and Chemical Engineering, Yantai University, Yantai 264005, China
}

\section{A R T I C L E I N F O}

\section{Article history:}

Received 12 November 2015

Received in revised form 13 January 2016

Accepted 14 January 2016

Available online 16 January 2016

\section{Keywords:}

Water-compatible

Molecularly imprinted polymers

Temperature responsive

Solid-phase extraction

Bisphenol A

Seawater

\begin{abstract}
A B S T R A C T
Versatile molecularly imprinted polymers (MIPs) have been widely applied to various sample matrices, however, molecular recognition in aqueous media is still difficult. Stimuli-responsive MIPs have received increasing attentions due to their unique feature that the molecular recognition is regulated by specific external stimuli. Herein, water-compatible temperature and magnetic dual-responsive MIPs (WC-TMMIPs) with hydrophilic brushes were prepared via reversible addition-fragmentation chain transfer precipitation polymerization for reversible and selective recognition and extraction of bisphenol A (BPA). Transmission electron microscopy (TEM), Fourier transform infrared spectrometer (FT-IR) and vibrating sample magnetometry (VSM) as characterization methods were used to examine the successful synthesis of polymers, and the resultant WC-TMMIPs showed excellent thermosensitivity and simple rapid magnetic separation. Controlled adsorption and release of BPA by temperature regulation were investigated systematically, and the maximum adsorption and removal efficiency toward BPA in aqueous solutions were attained at $35^{\circ} \mathrm{C}$ and $45^{\circ} \mathrm{C}$, respectively, as well as a good recoverability was exhibited with the precision less than $5 \%$ through five adsorption-desorption cycles. Phenolic structural analogs were tested and good recognition specificity for BPA was displayed. Accordingly, the WC-TMMIPs were employed as adsorbents for magnetic solid-phase extraction (MSPE) and packed SPE of BPA from seawater samples. Using the two modes followed by HPLC-UV determination, excellent linearity was attained in the range of $0.1-14.5 \mu \mathrm{M}$ and $1.3-125 \mathrm{nM}$, with low detection limits of $0.02 \mu \mathrm{M}$ and $0.18 \mathrm{nM}$, respectively. Satisfactory recoveries for spiked seawater samples were achieved ranging from $86.3-103.5 \%$ and 96.2-104.3\% with RSD within 2.12-4.33\%. The intelligent WC-TMMIPs combining water-compatibility, molecular recognition, magnetic separation, and temperature regulation proved potentially applicable for selective identification, controlled adsorption/release and high-efficiency enrichment/removal of trace targets in complicated aqueous media.
\end{abstract}

() 2016 Elsevier B.V. All rights reserved.

\section{Introduction}

Bisphenol A (BPA), an industrial chemical, is intensively used in the manufacture of many products such as engineering plastics, food cans and sealants. As an important phenolic environmental

\footnotetext{
* Corresponding author.

** Corresponding author.

E-mail addresses: dlyhy@dicp.ac.cn (H. You), lxchen@yic.ac.cn (L. Chen).
}

estrogen, its release into the surrounding environment can result in changing the function of the endocrine systems and consequently elicit negative health effects $[1,2]$. Within the last decades, various analytical and treatment methods have been developed for the recognition and determination of BPA in environmental waters and soil samples, including high performance liquid chromatography (HPLC) [3], gas chromatography (GC) [4], membrane separation technology [5], photo degradation [6], etc., which usually require solid-phase extraction (SPE) or liquid liquid extraction (LLE) steps for sample preparation and pretreatment. 
However, one common problem associated with conventional SPE is its low selectivity. Fortunately, molecularly imprinted polymers (MIPs) with a predetermined selectivity for target analytes, have been widely used as selective adsorbents, and relevant studies on antibiotics and estrogens have been increasingly reported [7-10]. For examples, Lv et al. [8] developed a molecularly imprinted organic-inorganic hybrid composite material for selective SPE of tetracyclines residues in milk. It was found that an enrichment factor of 18.8 along with a good sample clean-up was obtained under the optimized conditions by using HPLC determination. Afterward, Urraca et al. [9] proposed the synthesis of MIPs microbeads for the selective SPE of six fluoroquinolone antibiotics from chicken muscle samples. This optimized SPE method did not require time-consuming and labor-intensive extraction procedures and allowed the simultaneous determination of six fluoroquinolone antibiotics with good recoveries and low detection limits via HPLC-MS/MS. Mahony et al. [10] used hydroquinoneMIPs for SPE clean-up of BPA in milk samples through a rational design approach in which BPA was quantified by HPLC coupled with fluorescence detection. And the proposed method was capable of determining BPA in milk down to a detection limit of $1.32 \mu \mathrm{g} / \mathrm{kg}$. So, we expect to prepare MIPs-based materials for SPE to realize high-efficiency analytical determination of BPA in water samples.

In the meanwhile, stimuli-responsive MIPs (SR-MIPs) have attracted widespread concerns owing to the unique feature of molecular recognition specificity regulated by specific external stimuli [11]. They can respond to external stimuli signals such as magnetism, temperature, $\mathrm{pH}$ and light with a considerable change in their chain structure, surface structure or dissociation behavior, and so on. Excitingly, a number of SR-MIPs have been designed and synthesized for recognition and detection of BPA. For instances, Hiratsuka et al. [12] prepared magnetic MIPs (MMIPs) for BPA by a multi-step swelling and evaluated the retention and molecular recognition properties of MMIPs for BPA by HPLC. Besides, the determined concentration of BPA in river water samples was $68 \mathrm{ng} / \mathrm{L}$. It is found that magnetic SR-MIPs are mostly discussed while other type ones are few studied. Our group [13] has prepared thermosensitive MIPs (TMIPs) for selective recognition and controlled adsorption and release of BPA by the temperature regulation. The TMIPs were proved to be ideal candidates for SPE, and thereby the TMIPs-SPE was potentially applicable for highly efficient separation, extraction and determination of trace BPA in seawater and yogurt samples. Accordingly, dual-responsive MIPs (DR-MIPs) have attracted more and more attentions, e.g., photonic and magnetic DR-MIPs for high efficiency recognition and extraction of caffeine in cola, tap water and tea water [14]. and $\mathrm{pH}$ and temperature DR-MIPs for enhanced recognition toward ovalbumin in aqueous solutions [15].

Nevertheless, most MIPs are restricted to nonpolar or low polar solvents for their preparation and application. The presence of polar solvents, especially water, can generally destroy the interaction between functional monomers and template molecules, which would lead to low or invalid recognition in aqueous environments [7]. Currently, some water-compatible MIPs (WC-MIPs) have been prepared with improved recognition capability and removal efficiency in aqueous media $[16,17]$. Two major kinds of monomers are adopted, i.e., using specifically designed functional monomers like 2-hydroxyethyl methacrylate (HEMA) [3], $\beta$-cyclodextrin ( $\beta$ CD) [18] and acrylamide (AAm) [19], and functional co-monomers like the combination of $\beta-C D$ and $A A m[19,20]$ and the combination of N-isopropyl acrylamide (NIPAM), acrylic acid (AA) and azobenzene [21]. Among them, core-shell surface MIPs particles with hydrophilic polymer brushes are especially desirable. For example, Zhang et al. [22] demonstrated the controlled synthesis of pure water-compatible MIPs microspheres with ultrathin hydrophilic poly HEMA brushes and lightly cross-linked poly HEMA hydrogel layer via reversible addition-fragmentation chain transfer precipitation polymerization (RAFTPP), and investigated their molecular recognition ability in the pure aqueous solution. Yang et al. [23] synthesized photo-responsive surface MIPs on silica microspheres by surface polymerization using a water-soluble azobenzene containing 4-[(4-methacryloyloxy) phenylazo]benzene sulfonic acid as the functional monomer for the recognition and detection of BPA. However, to the best of our knowledge, magnetic and thermalresponsive DR-MIPs with water-compatible brushes have never been reported.

In this study, we prepared core-shell structured watercompatible temperature and magnetic responsive DR-MIPs (WCTMMIPs) with hydrophilic HEMA brushes by RAFTPP for selective recognition and extraction of $\mathrm{BPA}$, by taking $\mathrm{Fe}_{3} \mathrm{O}_{4}$ nanoparticles as magnetic core, $\mathrm{SiO}_{2}$ nanoparticles as shell materials, methacrylic acid (MAA) as functional monomer, and NIPAM as thermosensitive functional co-monomer. The morphology and composition of WCTMMIPs microspheres were characterized by transmission electron microscopy (TEM), Fourier-transform infrared spectroscopy (FTIR) and vibrating sample magnetometry (VSM). Binding property, thermal-responsive behavior, and magnetic separation ability were systematically explored through equilibrium binding analysis. And then their applications as adsorbents to magnetic SPE (MSPE) and packed SPE toward BPA from seawater samples were also investigated.

\section{Experimental}

\subsection{Materials and instruments}

BPA, phenol, $\beta$-estradiol, diethylstilbestrol and sodium acetate were obtained from Aladdin (Shanghai, China). Iron (III) chloride hexahydrate $\left(\mathrm{FeCl}_{3} \cdot 6 \mathrm{H}_{2} \mathrm{O}\right)$, ammonium hydroxide, ethylene glycol, tetrahydrofuran (THF) and polyethylene glycol were all obtained from Sinopharm Chemical Reagent Co., Ltd. (Shanghai, China). HPLC grade methanol and acetonitrile, and tetramethoxysilane (TEOS) were purchased from J\&K Scientific Ltd. (Beijing, China). MAA, ethyleneglycol dimethacylate (EGDMA), HEMA and NIPAM (99\%) were purchased from Sigma-Aldrich (Shanghai, China). 2,2-Azobisisobu-tyronitrile (AIBN) was purchased from Shanghai Chemical Plant (Shanghai, China). Ultrapure water was produced by a Cascada TM LS Ultrapure water system with the water outlet operating at $18.2 \mathrm{M} \Omega \mathrm{cm}$ (Pall Corp., USA) for aqueous solution preparation throughout the study. All solvents and chemicals were of analytic grade and used directly without further purification unless otherwise specified.

The morphological evaluation was performed by transmission electron microscopy (TEM, JEM-2100F). The characteristic functional groups were measured by a Fourier transform infrared spectrometer (FT-IR, Nicolet iS10, deduct air background). Magnetic property was measured by vibrating sample magnetometer (VSM, Lake Shore 7410, Beijing, China). The amounts of analytes were determined by UV-vis spectra (NanoDrop 2000/2000c, USA). Chromatographic analysis was carried out on a HPLC-UV instrument (LC-20AT, Shimadzu) under the optimized conditions: injection volume, $20 \mu \mathrm{L}$; mobile phase, acetonitrile/ultrapure water $(6: 4, \mathrm{v} / \mathrm{v})$; flow rate, $1.0 \mathrm{~mL} / \mathrm{min}$; DAD detection wavelength, $278 \mathrm{~nm}$; column, $250 \mathrm{~mm} \times 4.6 \mathrm{~mm} \mathrm{C}_{18}$ at $20^{\circ} \mathrm{C}$.

\subsection{Preparation of $\mathrm{Fe}_{3} \mathrm{O}_{4} @ \mathrm{SiO}_{2}$ with double bond}

Firstly, the monodispersed $\mathrm{Fe}_{3} \mathrm{O}_{4}$ nanoparticles were prepared by hydrothermal reaction according to a published procedure [24] with slight modifications. Briefly, $\mathrm{FeCl}_{3} \cdot 6 \mathrm{H}_{2} \mathrm{O}(1.35 \mathrm{~g})$ was dissolved in $40 \mathrm{~mL}$ ethylene glycol to form a clear solution and was stirred for 
$30 \mathrm{~min}$. Sodium acetate $(2.17 \mathrm{~g})$ was added with mechanical mixing vigorously for $10 \mathrm{~min}$ until dissolved. After then, polyethylene gly$\mathrm{col}(2 \mathrm{~g})$ was added into the above solution, being stirred for $30 \mathrm{~min}$ at $60^{\circ} \mathrm{C}$ and for $30 \mathrm{~min}$ at room temperature, respectively. Then the resulting clear yellowish solution was transferred to a Teflon lined stainless steel autoclave, sealed and heated to $200^{\circ} \mathrm{C}$. After reacting for $10 \mathrm{~h}$, the autoclave was cooled to room temperature. The collected magnetic products were purified in turn with ultrapure water and anhydrous ethanol for 6 times, and then were dispersed in ultrapure water for subsequent use.

Then, the $\mathrm{Fe}_{3} \mathrm{O}_{4} @ \mathrm{SiO}_{2}$ was prepared through the Stöber process [25]. Briefly, the above prepared $\mathrm{Fe}_{3} \mathrm{O}_{4}$ nanoparticles $(100 \mathrm{mg}$ ) were dispersed in the mixed solution of ethanol $(100 \mathrm{~mL})$ and ultrapure water $(20 \mathrm{~mL})$, being stirred for $10 \mathrm{~min}$, and then the solution was added into ammonium hydroxide to make the solution $\mathrm{pH}$ be alkaline, followed by the addition of TEOS $(1 \mathrm{~mL})$, reacting for $12 \mathrm{~h}$ at room temperature with continuously stirring. The resultant microspheres were washed by ethanol and ultrapure water followed by drying, which were denoted as $\mathrm{Fe}_{3} \mathrm{O}_{4} @ \mathrm{SiO}_{2}$.

Afterwards, the above dried $\mathrm{Fe}_{3} \mathrm{O}_{4} @ \mathrm{SiO}_{2}(1 \mathrm{~g})$ was dispersed in the mixture solution of ethanol $(75 \mathrm{~mL})$ and ultrapure water $(25 \mathrm{~mL})$, and the solution $\mathrm{pH}$ was adjusted to 4 using acetic acid. $\mathrm{KH}-570$ (25 mL, $1.2 \mathrm{wt} \%$ ethanol) was added to acidize solution under nitrogen protection, stirring for $24 \mathrm{~h}$ at room temperature. The products were washed by ethanol and ultrapure water for 3 times, respectively, and then were dried for further use, which were marked as $\mathrm{Fe}_{3} \mathrm{O}_{4} @ \mathrm{SiO}_{2}$ with double bond.

\subsection{Synthesis of chain transfer agent (CTA)}

CTA was synthesized according to our previous work [26]. Briefly, magnesium offals ( $1.5 \mathrm{~g})$, bromobenzene $(0.5 \mathrm{~mL})$, a grain of iodine and $20 \mathrm{~mL}$ absolutely dry THF were introduced into a threenecked flask under nitrogen atmosphere. Bromobenzene $(4.8 \mathrm{~mL})$ in THF $(30 \mathrm{~mL})$ was added dropwise with stirring until the brown of iodine disappeared. The reaction mixture was left to stand for $3 \mathrm{~h}$ to ensure a complete reaction of bromobenzene. Then the reaction solution was cooled down till room temperature, and carbon disulfide $(6 \mathrm{~mL})$ in THF $(20 \mathrm{~mL})$ was added dropwise, and the reaction was kept at that temperature for $2 \mathrm{~h}$. Benzyl bromide $(12 \mathrm{~mL})$ was then added, and the reaction mixture was heated to $40^{\circ} \mathrm{C}$ and kept for $2 \mathrm{~h}$. After cooling to room temperature, the solution was poured into $150 \mathrm{~mL}$ ultrapure water, and extracted by petroleum ether, followed by collecting and concentrating the organic phase. The residue was purified by chromatography using petroleum ether to produce cardinal liquid, namely CTA.

\subsection{Preparation of WC-TMMIPS}

TMMIPs without water-compatible brushes were firstly prepared via RAFTPP. Template (BPA, $0.1 \mathrm{mmol}$ ) and functional monomers (MAA/NIPAM, $0.6 \mathrm{mmol} / 0.6 \mathrm{mmol}$ ) were dissolved in acetonitrile $(60 \mathrm{~mL})$, and then the mixture was stored at $4{ }^{\circ} \mathrm{C}$ for pre-polymerization in dark for $12 \mathrm{~h}$. The prepared $\mathrm{Fe}_{3} \mathrm{O}_{4} @ \mathrm{SiO}_{2}$ with double bond was added into the pre-polymer solution, stirring evenly to room temperature in the case of growing temperature. When the temperature rose to $50^{\circ} \mathrm{C}$, cross-linker (EGDMA, $1.75 \mathrm{mmol}$ ), initiator (AIBN, $20 \mathrm{mg}$ ) and CTA $(60 \mu \mathrm{L})$ were added for polymerization over $24 \mathrm{~h}$ at $60^{\circ} \mathrm{C}$ under nitrogen protection. The obtained TMMIPs with dithioester groups were washed with methanol/acetic acid solution $(9: 1, v / v)$ to remove both the template molecules and residual monomers. Finally, they were dried to constant weight under vacuum at $40^{\circ} \mathrm{C}$, which were marked as TMMIPs.

Then, the WC-TMMIPs with grafted water-compatible brushes were prepared via surface-initiated RAFTPP according to the following procedure. The above prepared TMMIPs with dithioester groups $(150 \mathrm{mg})$ were dissolved in acetonitrile solution $(10 \mathrm{~mL})$, being mechanically stirred $15 \mathrm{~min}$ under nitrogen protection. When temperature rose to $70^{\circ} \mathrm{C}, \mathrm{HEMA}(13.5 \mathrm{mmol}), \mathrm{AIBN}(9 \mu \mathrm{mol})$ and CTA $(27 \mu \mathrm{mol})$ were added into the above solution, polymerizing for $24 \mathrm{~h}$. The resultant microspheres were named as WC-TMMIPs, and Fig. 1A(a) schematically shows the preparation process. Corresponding nonimprinted polymers, namely WC-TMNIPs, were prepared in the same manner as WC-TMMIPs but without adding template molecule in polymerization process.

\subsection{Adsorption experiments}

The effect of temperature $\left(20^{\circ} \mathrm{C}, 35^{\circ} \mathrm{C}\right.$, and $\left.45^{\circ} \mathrm{C}\right)$ on the adsorption was investigated by $10 \mathrm{mg}$ of WC-TMMIPs with $2 \mathrm{~mL}$ of $0.2 \mathrm{mM}$ BPA aqueous solution. Then the binding capacity ( $Q$ ) of WC-TMMIPs (or TMMIPs, WC-TMNIPs) for the template BPA was determined as follows. $10 \mathrm{mg}$ of WC-TMMIPs (or TMMIPs, WC-TMNIPs) were suspended in $2 \mathrm{~mL}$ of BPA aqueous solutions with various concentrations ranging from 0 to $0.5 \mathrm{mM}$. The series of mixtures were shaken for $12 \mathrm{~h}$ at $35^{\circ} \mathrm{C}$, and then the equilibrium concentrations of BPA were detected by UV-vis at $278 \mathrm{~nm}$. The binding amounts of BPA could be calculated by the following equation:

$Q_{e}=\frac{\left(C_{o}-C_{e}\right) V}{m}$

where $Q_{e}(\mathrm{mg} / \mathrm{g})$ is the equilibrium binding capacity of BPA, and $C_{o}(\mathrm{mM})$ and $C_{e}(\mathrm{mM})$ are the initial concentration and equilibrium adsorption concentrations of BPA, respectively. $V(\mathrm{~mL})$ is the volume of BPA solution and $m(\mathrm{~g})$ means the mass of polymers.

Meanwhile, for adsorption kinetics study, $20 \mathrm{mg}$ of WC-TMMIPs were suspended in $10 \mathrm{~mL}$ of $0.2 \mathrm{mM}$ BPA aqueous solution and shaken at $35^{\circ} \mathrm{C}$. The concentrations of BPA at a certain intervals $(30,60,120,180,240,300,420,540$ and $720 \mathrm{~min}$ ) were analyzed by the following equation:

$Q_{t}=\frac{\left(C_{o}-C_{t}\right) V}{m}$

where $Q_{t}$ is the temporal binding capacity of BPA, and $C_{o}(\mathrm{mM})$ and $C_{t}(\mathrm{mM})$ are the initial concentration and temporal adsorption concentration of BPA, respectively. $V(\mathrm{~mL})$ is the volume of BPA solution, and $m(\mathrm{~g})$ means the mass of polymers.

\subsection{Selectivity evaluation}

Phenol, $\beta$-estradiol and diethylstilbestrol were chosen as structural analogs to evaluate the selectivity of prepared polymers. $10 \mathrm{mg}$ of WC-TMMIPs or WC-TMNIPs were dispersed in $2 \mathrm{~mL}$ aqueous solution containing $0.2 \mathrm{mM}$ BPA and analogs, respectively. The binding capacities of BPA and structural analogs by WC-TMMIPs and WC-TMNIPs were compared.

\subsection{Preparation and analysis of seawater sample}

Surface seawater samples were collected from the Fisherman's Wharf of the Yellow Sea located in the coastal zone area of Yantai City, which were filtered with a $0.45 \mu \mathrm{m}$ PTFE syringe filters (Phenomenex, Los Angeles, CA, USA) to remove possible silt soil and sand grain, and then the extract aqueous solutions were collected and stored at $4{ }^{\circ} \mathrm{C}$ for use. Subsequently, magnetic SPE separation was performed with an exterior magnet, as illustrated in Fig. $1 \mathrm{~A}(\mathrm{~b})$. The specific process was as follows. $50 \mathrm{mg}$ of dry WC-TMMIPs were dispersed in $100 \mathrm{~mL}$ of the above-obtained filtered aqueous solutions at $35^{\circ} \mathrm{C}$, being shaken for $180 \mathrm{~min}$, and dealt with a magnet followed by HPLC-UV analysis. 

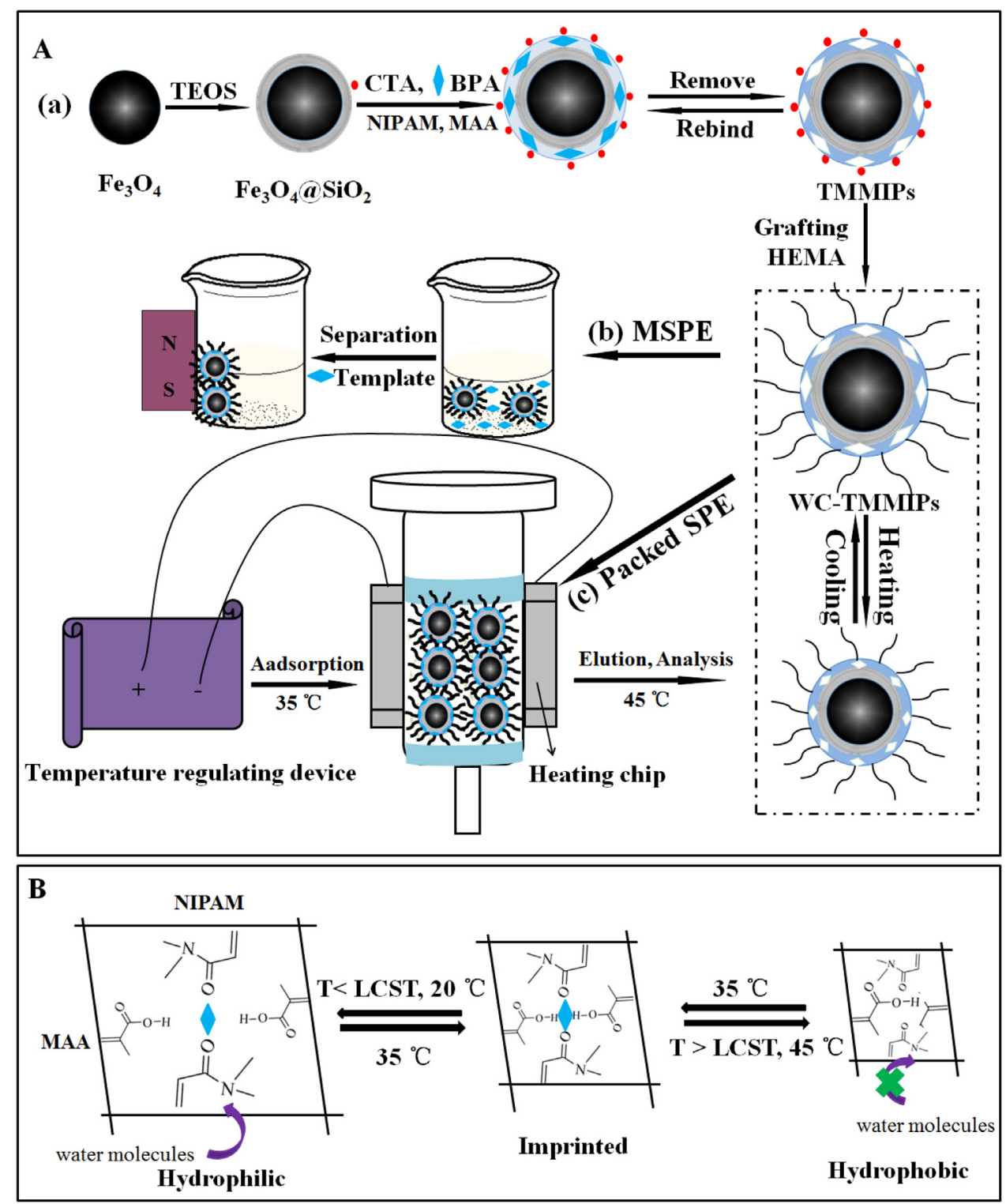

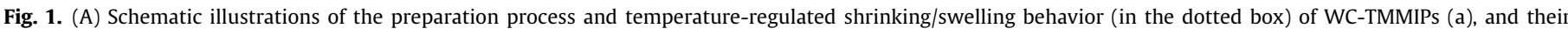

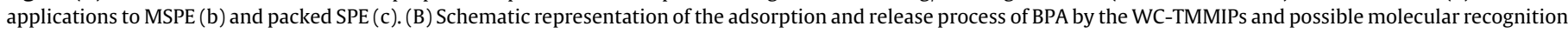
mechanism regulated by temperatures.

Also, the above-obtained filtered aqueous solutions of seawater samples were analyzed by using WC-TMMIPs as adsorbents of packed SPE at $35^{\circ} \mathrm{C}$ and $45^{\circ} \mathrm{C}$, respectively. Briefly, $400 \mathrm{mg}$ of dry WC-TMMIPs were packed into an empty $6 \mathrm{~mL}$ polypropylene home-built SPE cartridge comprising of a double-walled electric heating chip for controlling temperature, as shown in Fig. $1 \mathrm{~A}(\mathrm{c})$. Before the first use, the WC-TMMIPs in cartridges were sequentially washed with $3 \mathrm{~mL}$ of methanol, $3 \mathrm{~mL}$ of methanol/acetic acid (9:1, $\mathrm{v} / \mathrm{v}$ ), and $3 \mathrm{~mL}$ of ultrapure water. To find the optimum conditions, standard aqueous solution was chosen to evaluate the WC-TMMIPs as adsorbent in packed SPE. Afterwards, $10 \mathrm{~mL}$ of the filtered aqueous solutions of seawater samples were used as loading medium at $35^{\circ} \mathrm{C}, 5 \mathrm{~mL}$ of ultrapure water/methanol $(4: 6, \mathrm{v} / \mathrm{v})$ as washing solvent, and $5 \mathrm{~mL}$ of acetic acid/methanol $(1: 9, \mathrm{v} / \mathrm{v})$ as elution solvent at $45^{\circ} \mathrm{C}$. Finally, the eluent was evaporated using nitrogen blow, and then the residues were re-dissolved in $1 \mathrm{~mL}$ of ultrapure water for HPLC-UV analysis.

\section{Results and discussion}

\subsection{Preparation of WC-TMMIPS}

Fig. 1A schematically shows the preparation process of WCTMMIPs by RAFTPP (a), and their applications to MSPE (b) and conventional packed SPE (c). The synthesized $\mathrm{Fe}_{3} \mathrm{O}_{4}$ microspheres were capsulated by $\mathrm{SiO}_{2}$ on its surface, since $\mathrm{SiO}_{2}$ can not only be modified with various groups easily, but also prevent the oxidization and agglomeration of $\mathrm{Fe}_{3} \mathrm{O}_{4}$ [27]. After that, vinyl groups were introduced onto the surface of $\mathrm{Fe}_{3} \mathrm{O}_{4} @ \mathrm{SiO}_{2}$ for the following polymerization reaction with the functional monomers of MAA and NIPAM, template BPA and cross-linker EGDMA under AIBN initiation. The obtained TMMIPs were grafted with monomer HEMA to form hydrophilic polymerization brushes poly HEMA (Ref. Fig. 1A, the dotted box), and thereby the water-compatible TMMIPs, i.e., WC-TMMIPs, were prepared. 


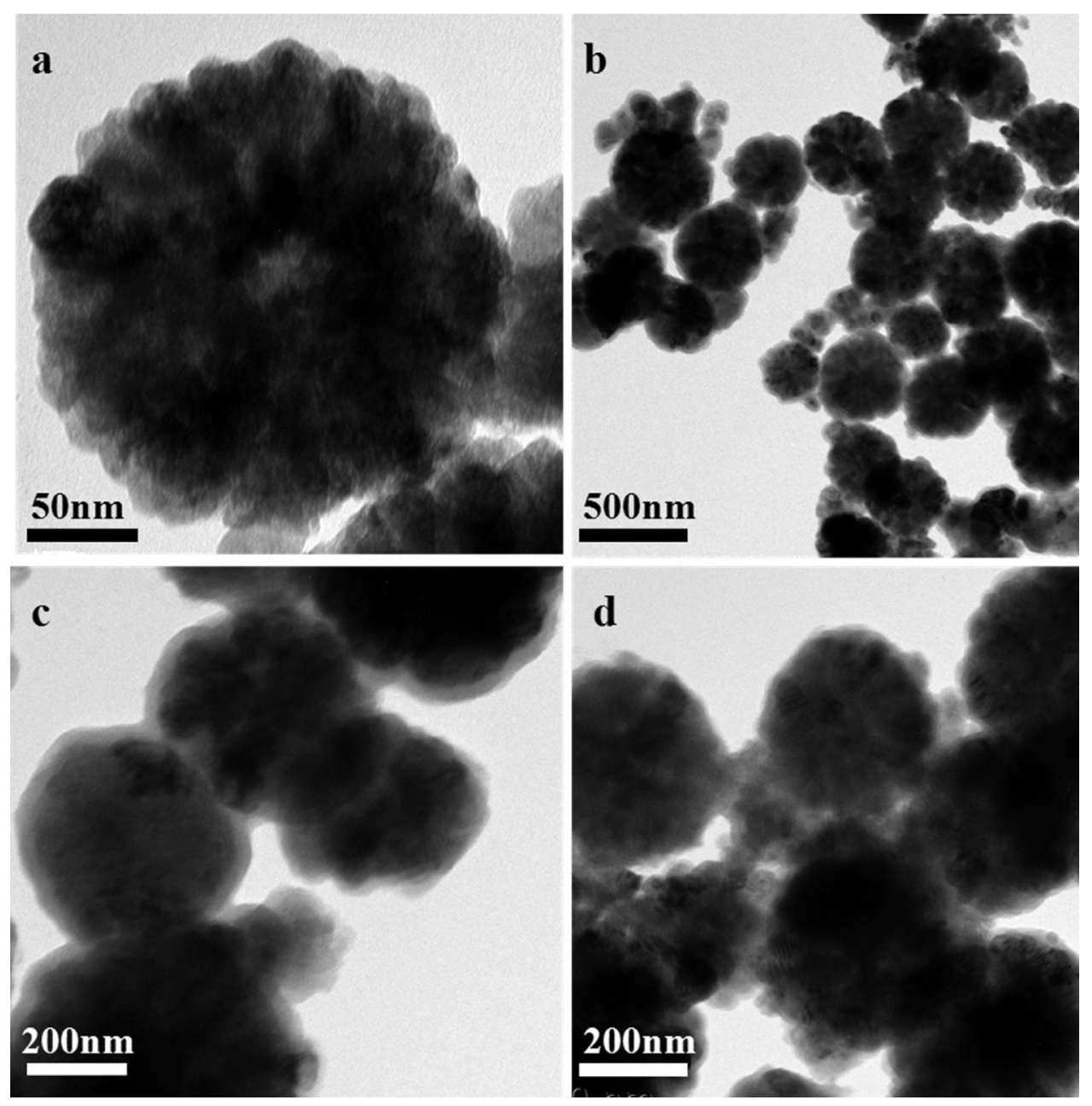

Fig. 2. TEM images of $\mathrm{Fe}_{3} \mathrm{O}_{4}$ (a), $\mathrm{Fe}_{3} \mathrm{O}_{4} @ \mathrm{SiO}_{2}$ (b), WC-TMMIPs (c) and WC-TMNIPs (d).

Consequently, the novel WC-TMMIPs with temperature and magnetic dual-responsive properties could recognize BPA via multiple-point hydrogen bonding [28]. Herein, MAA was used as functional monomer to recognize target molecule BPA. Besides, NIPAM, a widely used thermosensitive monomer, was adopted not only as functional co-monomer for more specific binding sites, but also as inert polymer backbone allowing for swelling and shrinking of the MIPs in response to temperature changes to realize recognition and release of BPA $[28,29]$. So, the synergy of MAA and NIPAM played an important role in preparation of the WC-TMMIPs, and would contribute greatly to their performances. Fig. 1B shows the whole process of recognition/adsorption and release of BPA. As seen, the reversible recognition/adsorption and release of target molecule BPA can be realized by changing temperature. Meanwhile, it was found that the NIPAM, as a wellknown temperature responsive polymer demonstrating a lower critical solution temperature (LCST) near $33^{\circ} \mathrm{C}$, had the property of a soluble (hydrophilic)-insoluble (hydrophobic) transition at the LCST of $35^{\circ} \mathrm{C}$ in this system. And it displays a coil (soluble) state when the solution temperature is below the LCST, as well as a collapsed (insoluble) state above the LCST because of a thermodynamic transition [30]. As illustrated in Fig. 1B, in the imprinting process at LCST such as $35^{\circ} \mathrm{C}$, BPA could well rebind with MAA and NIPAM by multiple-point hydrogen bonding, while the hydrogen bonding interactions would take some changes at other temperatures such as $20^{\circ} \mathrm{C}$ and $45^{\circ} \mathrm{C}$ owing to the thermally responsive behavior of NIPAM. Therefore, the as-prepared WC-TMMIPs could be employed for reversible thermo-regulated recognition, and controlled adsorption and release of template molecule BPA according to temperature changes, along with corresponding swelling and shrinking changes (Ref. Fig. 1A, the dotted box).

\subsection{Characterization of the WC-TMMIPS}

The morphological structures of $\mathrm{Fe}_{3} \mathrm{O}_{4}, \mathrm{Fe}_{3} \mathrm{O}_{4} @ \mathrm{SiO}_{2}$, WCTMMIPs and WC-TMNIPs were examined by TEM, as shown in Fig. 2. As seen, the diameter of $\mathrm{Fe}_{3} \mathrm{O}_{4}$ was about $300 \mathrm{~nm}$ (Fig. 2a). Meanwhile, $5 \mathrm{~nm}$ thick $\mathrm{SiO}_{2}$ layer was uniformly coated on $\mathrm{Fe}_{3} \mathrm{O}_{4}$ (Fig. 2b). As shown in Fig. 2c, the WC-TMMIPs microspheres were uniform in size distribution, and the thickness of the imprinting shell layer was about $10 \mathrm{~nm}$, which suggested that a large number of recognition sites were well distributed on the surface of $\mathrm{Fe}_{3} \mathrm{O}_{4} @ \mathrm{SiO}_{2}$. However, the WC-TMNIPs seemed not uniform in size distribution (Fig. 2d). The TEM images proved the core-shell structured MIPs were prepared.

The chemical structures of $\mathrm{Fe}_{3} \mathrm{O}_{4}, \mathrm{Fe}_{3} \mathrm{O}_{4} @ \mathrm{SiO}_{2}$, TMMIPs and WC-TMMIPs were investigated by FT-IR, as displayed in Fig. 3. The strong absorption peak at $580 \mathrm{~cm}^{-1}$ could be attributed to the stretching vibration of the $\mathrm{Fe}-\mathrm{O}$ (Fig. 3a), being the characteristic absorption peak of $\mathrm{Fe}_{3} \mathrm{O}_{4}$. The peaks at $800 \mathrm{~cm}^{-1}$ and $1090 \mathrm{~cm}^{-1}$ might well result from the symmetric and asymmetric stretching vibration of $\mathrm{Si}-\mathrm{O}$, which indicated $\mathrm{SiO}_{2}$ shells were successfully coated on the surface of $\mathrm{Fe}_{3} \mathrm{O}_{4}$ (Fig. 3b). The characteristic peak of $\mathrm{C}-\mathrm{H}$ at $2930 \mathrm{~cm}^{-1}$ could be ascribed to the $\mathrm{KH}-570$ modification on silica shells. The peak at $1710 \mathrm{~cm}^{-1}$ for the $\mathrm{C}=\mathrm{O}$ of EDGMA showed that MIP layers were grafted onto the surface of the $\mathrm{Fe}_{3} \mathrm{O}_{4} @ \mathrm{SiO}_{2}$ 


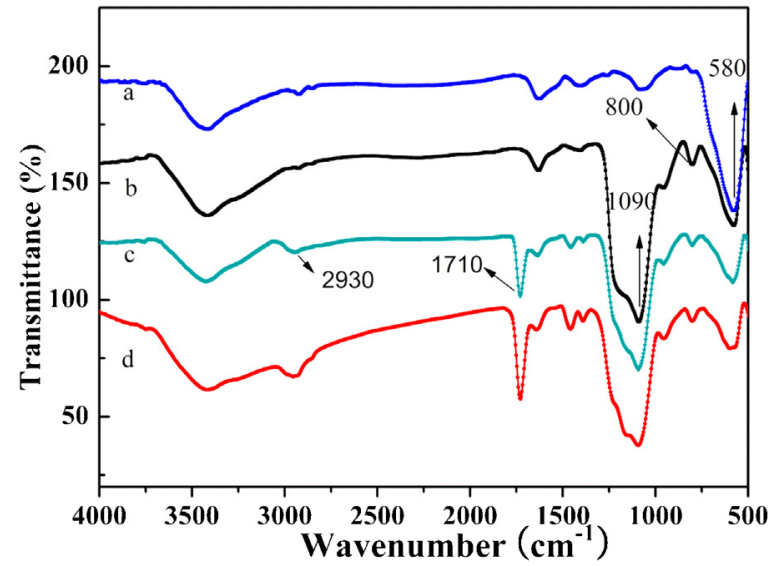

Fig. 3. FT-IR spectra of $\mathrm{Fe}_{3} \mathrm{O}_{4}$ (a), $\mathrm{Fe}_{3} \mathrm{O}_{4} @ \mathrm{SiO}_{2}$ (b), TMMIPs (c) and WC-TMMIPs (d).

support (Fig. 3c), which proved large amounts of recognition sites for the template were obtained in the TMMIPs. Interestingly, the intensity became larger of the peak at $1710 \mathrm{~cm}^{-1}$ in Fig. 3d, probably owing to the contribution of poly HEMA, and this proved polymer brush was grafted successfully. All the results of FT-IR confirmed that the WC-TMMIPs were successfully prepared by RAFTPP.

The magnetic property was measured by VSM, and Fig. S1 shows the magnetic hysteresis loops of $\mathrm{Fe}_{3} \mathrm{O}_{4}, \mathrm{Fe}_{3} \mathrm{O}_{4} @ \mathrm{SiO}_{2}$ and WC-TMMIPs. As seen, the three materials presented similar general shape loops, and the saturation magnetization value of WCTMMIPs was low (38.3 emu/g) compared with that of $\mathrm{Fe}_{3} \mathrm{O}_{4} @ \mathrm{SiO}_{2}$ $(58.3 \mathrm{emu} / \mathrm{g})$ and $\mathrm{Fe}_{3} \mathrm{O}_{4}(78.6 \mathrm{emu} / \mathrm{g})$. The magnetic saturation would gradually decrease with the increase of the coating layer thickness. Still, the prepared WC-TMMIPs were proved magnetically responsive. Accordingly, the homogeneously dispersed WC-TMMIPs could go straight towards the magnet and adhere to the inner side wall of the vials when the external magnetic field was used, as depicted in Fig. 1A(b), showing a fast and simple magnetic separation.

\subsection{Thermoresponsive property of WC-TMMIPs}

The effects of temperature on the BPA adsorption capacity were investigated for WC-TMMIPs at different temperatures such as $20^{\circ} \mathrm{C}, 35^{\circ} \mathrm{C}$ and $45^{\circ} \mathrm{C}$. As observed from Fig. $4 \mathrm{~A}$, the adsorption amounts of WC-TMMIPs at $35^{\circ} \mathrm{C}$ around LCST $\left(33^{\circ} \mathrm{C}\right)$ were much higher than that at $20^{\circ} \mathrm{C}$ and $45^{\circ} \mathrm{C}$, as well as the adsorption amounts at $20^{\circ} \mathrm{C}$ were higher than that at $45^{\circ} \mathrm{C}$. This result suggested the WC-TMMIPs had thermal-switchable effect on BPA with the change of temperature because of the borderline hydrophobic nature of the NIPAM units. That is, both hydrophilic amide groups and borderline hydrophobic isopropyl groups in the NIPAM units might cause a reversible volume transition of WC-TMMIPs between the collapsed and swollen phases under temperature stimuli.

Noticeably, the maximum adsorption amounts were attained at the temperature around LCST (Fig. 4A), $35^{\circ} \mathrm{C}$, indicating the cavity of WC-TMMIPs reached the imprinting state [31,32]. The schematic illustration of temperature-regulated adsorption/release of the WC-TMMIPs can be found in Fig. 1B. As seen, when the temperature was decreased to $20^{\circ} \mathrm{C}$ below the LCST, the WC-TMMIPs became highly hydrophilic and BPA molecules preferably approached the WC-TMMIPs and were captured. So, adsorption dominated at the temperature of $20^{\circ} \mathrm{C}$. In the meanwhile, water molecules easily diffused into the WC-TMMIPs and resulted in the polymers' swelling and increase of the distance between BPA and polymer matrix, and hence decreased the intermolecular multiple-point hydrogenbonding interaction between BPA and MAA, leading to release of
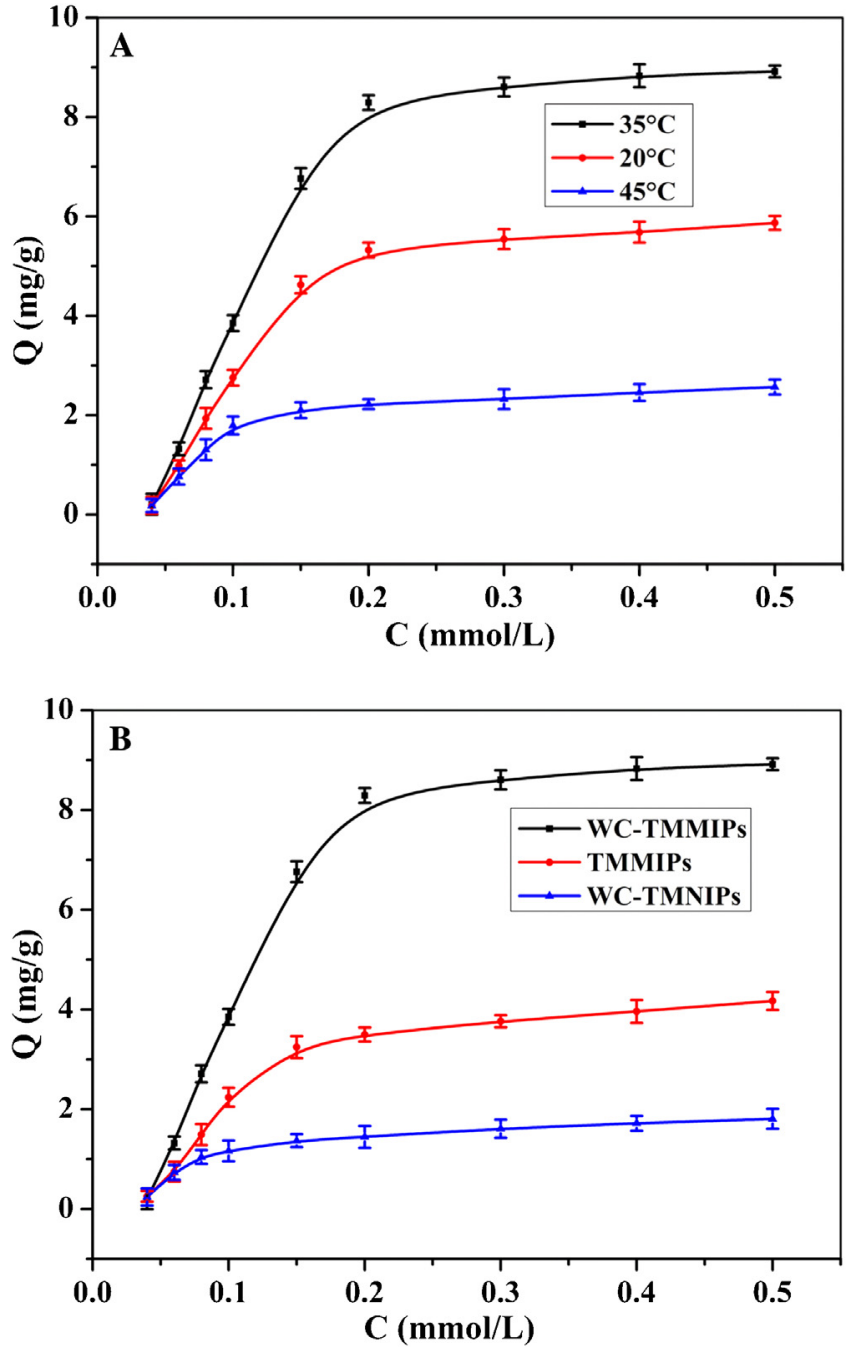

Fig. 4. Equilibrium adsorption isotherms of WC-TMMIPs for BPA at 20,35 and $45^{\circ} \mathrm{C}$ (a). Equilibrium adsorption curves of WC-TMMIPs/WC-TMNIPs and TMMIPs for BPA at $35^{\circ} \mathrm{C}(\mathrm{b})$

BPA. On balance, the adsorption capacity was lower than that at $35^{\circ} \mathrm{C}$. However, on the other hand, when the solution temperature was increased to $45^{\circ} \mathrm{C}$ above the LCST, water molecules were greatly expelled from the polymer interior, and then the volume of polymer had a drastic decrease, leading to a contractive state. Therefore, the hydrogen-bonding interaction was suppressed, and the polymer became hydrophobic. Consequently, the resultant collapsed WC-TMMIPs caused a deformation of the imprinted cavities, as observed from Fig. 1B, and thereby most of BPA molecules were released from the WC-TMMIPs and brought about the less adsorption amounts of BPA [32]. Hence, the released amounts of BPA at $45^{\circ} \mathrm{C}$ were much higher than that at $35^{\circ} \mathrm{C}$ and $20^{\circ} \mathrm{C}$ since the WCTMMIPs became shrank, hydrophobic and the hydrogen bonding was disturbed. All these results indicated that the WC-TMMIPs had good temperature sensitivity, which is important for adsorption and release of the template in binding and extraction. Therefore, $35^{\circ} \mathrm{C}$ was chosen as the optimum temperature to rebind BPA and conduct the following experiments, while $45^{\circ} \mathrm{C}$ was selected for the release of BPA.

Since the WC-TMMIPs had good thermosensitivity, showing reversible swelling and shrinking along with environmental temperature change, their reusability was also further investigated, which has a great cost benefit on extending their applications. The adsorbed BPA was fully released when the temperature of 
aqueous solution was changed to $45^{\circ} \mathrm{C}$, and then the free BPA was fully rebound when the temperature was changed to $35^{\circ} \mathrm{C}$. The temperature-regulated elution offered significant superiorities to the conventional organic-solvent elution, such as low solvent consumption, short process, rapid and easy operation, and environmental friendliness. Results indicated that the WCTMMIPs were excellently reusable, due to the recoverability of five adsorption-desorption cycles with the relative standard deviations (RSDs) less than $5 \%$.

After then, the batch rebinding experiments of WCTMMIPs/WC-TMNIPs and TMMIPs were carried out for BPA in a range of $0-0.5 \mathrm{mM}$ in the pure water solutions at $35^{\circ} \mathrm{C}$. From Fig. 4B, it can be clearly seen that both WC-TMMIPs and TMMIPs could bind more template molecules than WC-TMNIPs. The results suggested selective binding sites were present in the WC-TMMIPs and TMMIPs, while there were no specific adsorption property of WC-TMNIPs since the possible adsorption and release followed a physical process. As observed, the equilibrium adsorption capacity of WC-TMMIPs increased remarkably from 0.04 to $0.2 \mathrm{mM}$, and then became saturation with the capacity of $8.292 \mathrm{mg} / \mathrm{g}(36.3 \mu \mathrm{mol} / \mathrm{g})$ at $0.2 \mathrm{mM}$. Moreover, it was noticed that the maximum binding capacity of WC-TMMIPs was about 2 times of TMMIPs, suggesting the grafting brushes played an important role. The TMMIPs were grafted with hydrophilic HEMA brushes via surface-initiated RAFTPP, and thereby dimension-tunable WCTMMIPs could be attained with advanced architectures containing grafting via controlling the chain length realized by stopping the polymerization at a predetermined time [33]. The poly HEMA brushes not only significantly improved the surface hydrophilicity and enhanced water-compatibility of the TMMIPs, but also acted as a protective layer lest the other interferent blocking imprinting sites, and thus enabled the TMMIPs to function preferably in aqueous solutions. In addition, it was demonstrated that the grafting of polymer brushes is an efficient approach for the preparation of water-compatible MIPs, and accordingly enhances their selective recognition ability and adsorption capacity in aqueous media. Therefore, the WC-TMMIPs had more recognition sites and could rebind more BPA in aqueous media than TMMIPs without HEMA.

\subsection{Kinetic binding properties and selectivity of the WC-TMMIPS}

The adsorption kinetics of BPA onto the WC-TMMIPs was investigated at the maximum adsorption temperature of $35^{\circ} \mathrm{C}$. As shown in Fig. 5A, the adsorption processes were time dependent and the adsorption capacity of WC-TMMIPs increased rapidly with time in the initial $300 \mathrm{~min}$. Then, the adsorption rate became slow and reached equilibrium at last. In contrast, the WC-TMNIPs had much lower adsorption capacities, although they showed similar trend on the BPA adsorption. This was very likely because of the non-specific adsorption of NIPs owing to lacking suitable imprinting cavities. However, there were numerous imprinting sites in the WC-TMMIPs, leading to specific adsorption, and hence they produced higher adsorption capacity. So, the presence of complementary cavities to BPA in the MIPs is crucial for the specific adsorption.

To further evaluate the selectivity of the WC-TMMIPs, three phenolic structural analogs of phenol, $\beta$-estradiol and diethylstilbestrol were adopted. As seen from Fig. 5B, the adsorption capacities of WCTMMIPs for BPA, phenol, $\beta$-estradiol and diethylstilbestrol were $8.30,3.77,2.85$ and $1.92 \mathrm{mg} / \mathrm{g}$, respectively, which showed that the WC-TMMIPs had the highest molecular recognition ability toward BPA. In the meanwhile, the selectivity of WC-TMNIPs was low and the adsorption capacities for the four compounds were nearly similar, since there were no tailor-made recognition sites in the NIPs.
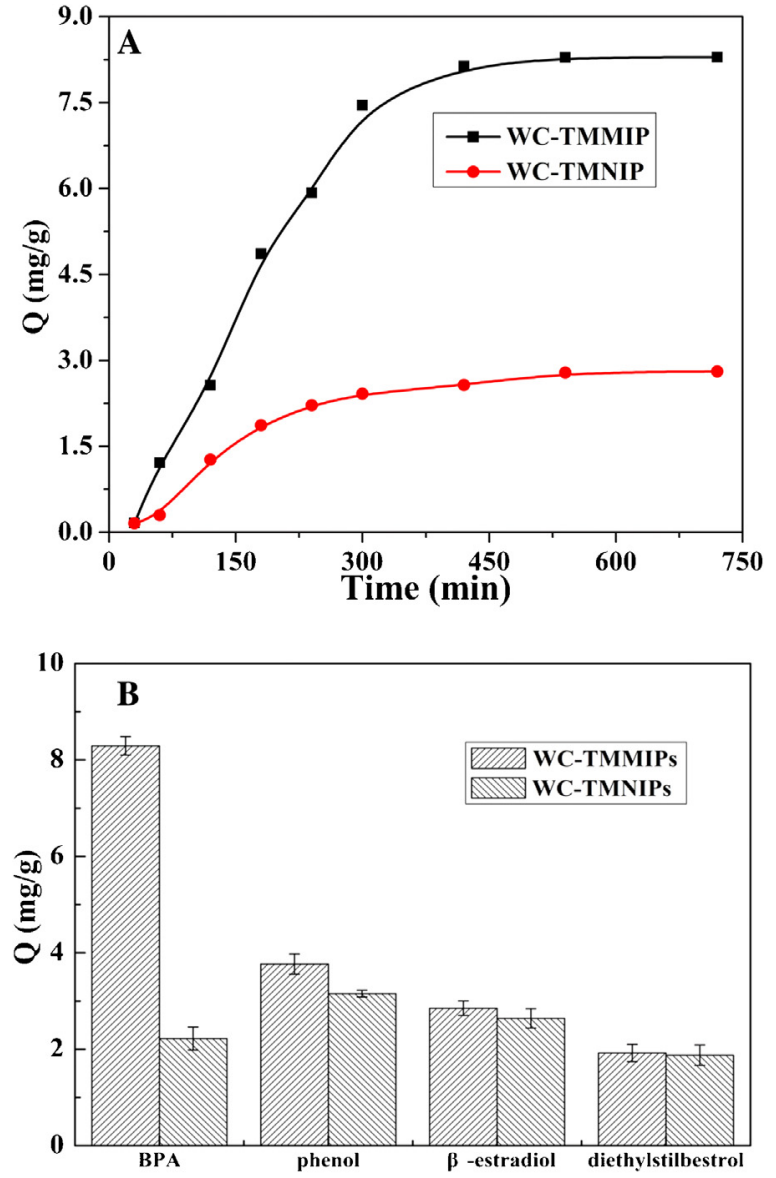

Fig. 5. Kinetic adsorption curves of WC-TMMIPs/WC-TMNIPs for BPA at $35^{\circ} \mathrm{C}(\mathrm{A})$. Selective adsorption of WC-TMMIPs and WC-TMNIPs for BPA, phenol, $\beta$-estradiol and diethylstilbestrol (B). Experimental conditions: $30 \mathrm{mg}$ WC-TMMIPs adsorbent, shaking time $3 \mathrm{~h}$.

Table 1

Recovery of BPA in seawater samples using WC-TMMIPs as adsorbents for MSPE and packed SPE at $35^{\circ} \mathrm{C}$ followed by UV-vis/HPLC determination $(n=3)$.

\begin{tabular}{lcccl}
\hline Procedure & Spiked & Determined & Recovery (\%) & RSD (\%) \\
\hline MSPE $(\mu \mathrm{M})$ & 2 & 2.1 & 91.2 & 4.32 \\
& 5 & 4.7 & 86.3 & 2.96 \\
& 10 & 10.8 & 103.5 & 3.79 \\
WC-TMMIPs-SPE & 10 & 10.1 & 96.2 & 2.12 \\
$(\mathrm{nM})$ & 50 & 52.1 & 104.3 & 4.33 \\
& 100 & 102.3 & 102.5 & 3.41 \\
\hline
\end{tabular}

So, the WC-TMMIPs exhibited high selectivity and molecular recognition ability toward BPA.

\subsection{Applications of the WC-TMMIPs as adsorbents for MSPE and packed SPE to seawater samples}

According to the above results, the as-prepared WC-TMMIPs could be utilized as adsorbents for selective recognition and extraction of BPA. In order to evaluate the practical applicability of the WC-TMMIPs, seawater samples were treated by the two modes of MSPE and packed SPE. The utilization of solvent elution and temperature-regulated release ensured effective recognition and extraction of BPA. As listed in Table 1, high recoveries of $86.3-104.3 \%$ with RSDs of $2.12-4.33 \%$ were obtained for the seawater samples, spiked with BPA at three concentration levels. Meanwhile, as seen in Fig. 6, BPA was specifically identified and remarkably concentrated by SPE packed with WC-TMMIPs, which 


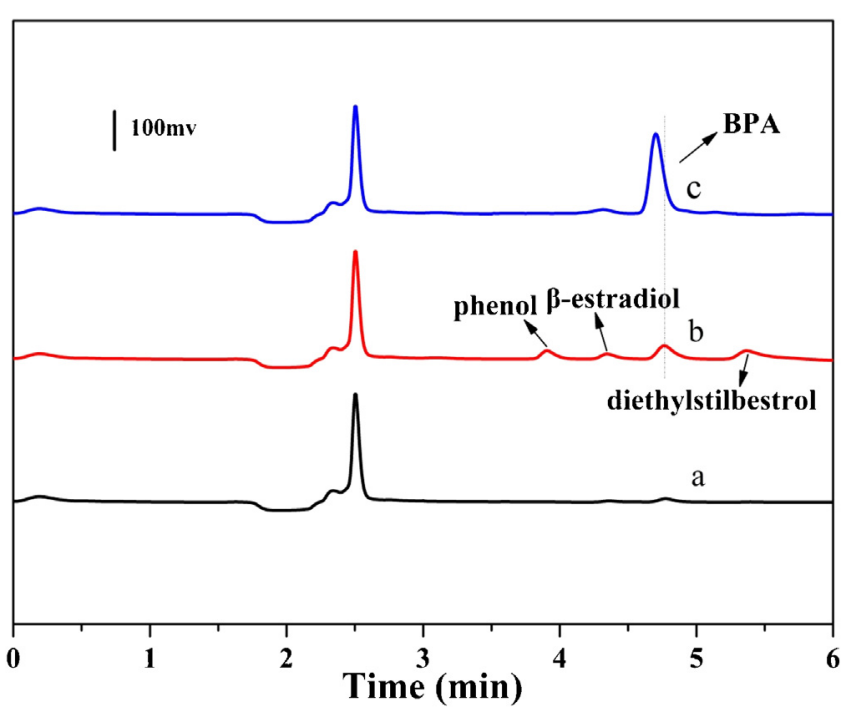

Fig. 6. HPLC chromatograms of seawater without spiking (a), and seawater spiked with BPA, phenol, $\beta$-estradiol and diethylstilbestrol individual at $100 \mathrm{nM}$ without extraction (b), and with WC-TMMIPs-SPE (c) at $45^{\circ} \mathrm{C}$. Experimental conditions: $5 \mathrm{~mL}$ spiked solutions; $400 \mathrm{mg}$ WC-TMMIPs; wash solution: $5 \mathrm{~mL}$ of ultrapure water/methanol (4:6, v/v); elution solution: $5 \mathrm{~mL}$ of acetic acid/methanol $(1: 9, \mathrm{v} / \mathrm{v})$; mobile phase: acetonitrile/ultrapure water $(6: 4, \mathrm{v} / \mathrm{v})$; flow rate: $1.0 \mathrm{~mL} / \mathrm{min}$; room temperature; DAD detection: $278 \mathrm{~nm}$; injection volume: $20 \mu \mathrm{L}$.

was attributed to the fact that the MIPs had high imprinting efficiency, so the matrix interferences could be drastically reduced and trace BPA could be pre-concentrated. The results indicated that the WC-TMMIPs were ideal candidates for SPE, and thereby the WC-TMMIPs-SPE was potentially applicable for highly efficient separation, extraction and determination of trace BPA in real aqueous samples.

In the seawater samples, low limits of detection (LOD, $S / N=3$ ) for BPA by using MSPE and packed SPE were achieved to be $0.02 \mu \mathrm{M}$ and $0.18 \mathrm{nM}$, respectively. The values can reach the required detectability for BPA in environmental water safety monitoring. Noticeably, the LOD provided by packed SPE was much lower than that of MSPE, indicating its higher enrichment ability. More comparisons can be found in Table S1, for example, the MSPE provided less adsorbent consumption and higher adsorption efficiency, while packed SPE provided shorter adsorption time and lower sample consumption. All these results revealed that the MSPE was more suitable for simple, rapid, recycled separation and adsorption of BPA, while packed SPE was more favorable to BPA enrichment and matrices elimination.

\subsection{Method performance comparison}

Method performance of this developed WC-TMMIPs packed SPE method toward BPA in aqueous media was compared with that of some reported MIPs based methods [13,23,34-41], as listed in Table S2. As seen from the table, this present method demonstrates higher sensitivity and higher adsorption capacity in aqueous solutions than that reported [13,23,36,37,39,41]. Two kinds of MIPs offer higher adsorption capacities in organic solvents but not in aqueous media $[35,40]$. and the LOD is also higher. The adsorption capacity of one kind of WC-MIPs prepared by RAFT using 2-acrylamido-2-methylpropanesulfonic acid as water-compatible functional monomer [34] is lower than the WC-TMMIPs. Hence, overall, the superiority of our developed WC-TMMIPs-SPE method is obvious on account of the low LODs and high adsorption capacities, and aqueous applicability, and moreover, it is simple, rapid and eco-friendly when using the magnetic separation and temperature regulation.

\section{Conclusions}

In conclusion, a new type of WC-TMMIPs with good water compatibility and sensitive dual stimuli-responsiveness were smartly prepared by facile RAFTPP via synergy of dual functional monomers, and they were successfully applied to reversible and selective recognition, controlled adsorption/release and effective enrichment/removal of BPA in seawater samples. The WC-TMMIPs demonstrated excellent features, such as high adsorption capacity, fast rebinding kinetics, good selectivity and stability, as well as simple rapid magnetic separation and excellent temperature responsive properties. The WC-TMMIPs were utilized for MSPE with direct dispersion in solutions and for conventional SPE with column packing, respectively, suggesting MSPE was more suitable for rapid adsorption while packed SPE was more favorable to higheffective enrichment. The WC-TMMIPs were proved ideal sorbents candidates for selective recognition and extraction of trace levels BPA from complicated environmental water samples by simple stimuli responsive regulation. We believe the present study can provide a general promising way to explore diverse SR-MIPs for versatile utilizations besides environmental friendly sample pretreatment. This aspect is currently in progress.

\section{Acknowledgments}

This work was financially supported by the National Natural Science Foundation of China (21477160, 21275158), and the National Key Scientific Instrument and Equipment Development Project (2013YQ17052506).

\section{Appendix A. Supplementary data}

Supplementary data associated with this article can be found, in the online version, at http://dx.doi.org/10.1016/j.chroma.2016.01. 040.

\section{References}

[1] Y.Q. Huang, C.K.C. Wong, J.S. Zheng, H. Bouwman, R. Barra, B. Wahlström, L. Neretin, M.H. Wong, Bisphenol A (BPA) in China: a review of sources, environmental levels, and potential human health impacts, Environ. Int. 42 (2012) 91-99.

[2] Y.Y. Wen, J.H. Li, J.S. Liu, W.H. Lu, J.P. Ma, L.X. Chen, Dual cloud point extraction coupled with hydrodynamic-electrokinetic two-step injection followed by micellar electrokinetic chromatography for simultaneous determination of trace phenolic estrogens in water samples, Anal. Bioanal. Chem. 405 (2013) 5843-5852.

[3] M. Hadjicharalampous, V.F. Samanidou, Analysis of organic components released from dental resin composites in saliva and other biological fluids using chromatographic techniques, Curr. Org. Chem. 14 (2010) 2329-2336.

[4] Y. Deceuninck, E. Bichon, P. Marchand, C.Y. Boquien, A. Legrand, C. Boscher, J.P. Antigac, B.L. Bizec, Determination of bisphenol A and related substitutes/analogues in human breast milk using gas chromatography-tandem mass spectrometry, Anal. Bioanal. Chem. 407 (2015) 2485-2497.

[5] M.S. Silva, R. Viveiros, M.B. Coelho, A.A. Aguiar-Ricardo, T. Casimiro, Supercritical $\mathrm{CO}_{2}$-assisted preparation of a PMMA composite membrane for bisphenol A recognition in aqueous environment, Chem. Eng. Technol. 68 (2012) 94-100

[6] M.Y. Mehr, W.D. van Driel, K.M.B. Jansen, P. Deeben, M. Boutelje, G.Q. Zhang, Photodegradation of bisphenol A polycarbonate under blue light radiation and its effect on optical properties, Opt. Mater. 35 (2013) 504-508.

[7] L.X. Chen, S.F. Xu, J.H. Li, Recent advances in molecular imprinting technology: current status, challenges and highlighted applications, Chem. Soc. Rev. 40 (2011) 2922-2942.

[8] Y.K. Lv, L.M. Wang, L. Yang, C.X. Zhao, H.W. Sun, Synthesis and application of molecularly imprinted poly(methacrylic acid)-silica hybrid composite material for selective solid-phase extraction and high-performance liquid chromatography determination of oxytetracycline residues in milk, J. Chromatogr. A 1227 (2012) 48-53.

[9] J.L. Urraca, M. Castellari, C.A. Barrios, M.C. Moreno-Bondi, Multiresidue analysis of fluoroquinolone antimicrobials in chicken meat by molecularly imprinted solid-phase extraction and high performance liquid chromatography, J. Chromatogr. A 1343 (2014) 1-9. 
[10] J.O. Mahony, M. Moloney, M. Mccormack, L.A. Nicholls, B. Mizaikoff, M. Danaher, Design and implementation of an imprinted material for the extraction of the endocrine disruptor bisphenol A from milk, J. Chromatogr. B 931 (2013) 164-169.

[11] S.F. Xu, H.Z. Lu, X.W. Zheng, L.X. Chen, Stimuli-responsive molecularly imprinted polymers: versatile functional materials, J. Mater. Chem. C 1 (2013) 4406-4422.

[12] Y. Hiratsuka, N. Funaya, H. Matsunaga, J. Haginaka, Preparation of magnetic molecularly imprinted polymers for bisphenol A and its analogues and their application to the assay of bisphenol A in river water, J. Pharm. Biomed. 75 (2013) 180-185.

[13] R.C. Dong, J.H. Li, H. Xiong, W.H. Lu, H.L. Peng, L.X. Chen, Thermosensitive molecularly imprinted polymers on porous carriers: preparation, characterization and properties as novel adsorbents for bisphenol A, Talanta 130 (2014) 182-191.

[14] S.F. Xu, J.H. Li, X.L. Song, J.S. Liu, H.Z. Lu, L.X. Chen, Photonic and magnetic dual responsive molecularly imprinted polymers: preparation, recognition characteristics and properties as a novel sorbent for caffeine in complicated samples, Anal. Methods 5 (2013) 124-133.

[15] F.X. Gao, X.L. Zhao, X.W. He, W.Y. Li, Y.K. Zhang, A pH and temperature dual-responsive macroporous molecularly imprinted cryogel for enhanced recognition capability towards ovalbumin, Anal. Methods 5 (2013) 6700-6708.

[16] H.Q. Zhang, Water-compatible molecularly imprinted polymers: promising synthetic substitutes for biological receptors, Polymer 55 (2014) 699-714.

[17] Y.L. Hu, J.L. Pan, K.G. Zhang, H.X. Lian, G.K. Li, Novel applications of molecularly-imprinted polymers in sample preparation, Trend. Anal. Chem. 43 (2013) 37-52.

[18] Z.F. Xu, L. Xu, D.Z. Kuang, F.X. Zhang, J.Q. Wang, Exploiting $\beta$-cyclodextrin as functional monomer in molecular imprinting for achieving recognition in aqueous media, Mater. Sci. Eng. C 28 (2008) 1516-1521.

[19] L. Oin, X.W. He, W.Y. Li, Y.K. Zhang, Molecularly imprinted polymer prepared with bonded $\beta$-cyclodextrin and acrylamide on functionalized silica gel for selective recognition of tryptophan in aqueous media, J. Chromatogr. A 1187 (2008) 94-102.

[20] W. Zhang, L. Qin, X.W. He, W.Y. Li, Y.K. Zhang, Novel surface modified molecularly imprinted polymer using acryloyl- $\beta$-cyclodextrin and acrylamide as monomers for selective recognition of lysozyme in aqueous solution, J. Chromatogr. A 1216 (2009) 4560-4567.

[21] G.Q. Pan, Y. Ma, Y. Zhang, X.Z. Guo, C.X. Li, H.Q. Zhang, Controlled synthesis of water-compatible molecularly imprinted polymer microspheres with ultrathin hydrophilic polymer shells via surface-initiated reversible addition-fragmentation chain transfer polymerization, Soft Matter 7 (2011) 8428-8439.

[22] M. Zhao, C. Zhang, Y. Zhang, X.Z. Guo, H.S. Yan, H.Q. Zhang, Efficient synthesis of narrowly dispersed hydrophilic and magnetic molecularly imprinted polymer microspheres with excellent molecular recognition ability in a real biological sample, Chem. Commun. 50 (2014) 2208-2210.

[23] Y.Z. Yang, Q. Tang, C.B. Gong, X.B. Ma, J.D. Peng, M.H.W. Lam, Ultrasensitive detection of bisphenol $\mathrm{A}$ in aqueous media using photoresponsive surface molecular imprinting polymer microspheres, New J. Chem. 38 (2014) $1780-1788$

[24] R.X. Gao, X. Kong, X. Wang, X.W. He, L.X. Chen, Y.K. Zhang, Preparation and characterization of uniformly sized molecularly imprinted polymers functionalized with core-shell magnetic nanoparticles for the recognition and enrichment of protein, J. Mater. Chem. 21 (2011) 17863-17871.
[25] B. Luo, X.J. Song, F. Zhang, A. Xia, W.L. Yang, J.H. Hu, C.C. Wang, Multi-functional thermosensitive composite microspheres with high magnetic susceptibility based on magnetite colloidal nanoparticle clusters, Langmuir 26 (2010) 1674-1679.

[26] S.F. Xu, J.H. Li, L.X. Chen, Molecularly imprinted polymers by reversible addition-fragmentation chain transfer precipitation polymerization for preconcentration of atrazine in food matrices, Talanta 85 (2011) 282-289.

[27] X.Y. Guo, F.F. Mao, W.J. Wang, Y. Yang, Z.M. Bai, Sulfhydryl-modified $\mathrm{Fe}_{3} \mathrm{O}_{4} @ \mathrm{SiO}_{2}$ core/shell nanocomposite: synthesis and toxicity assessment in vitro, ACS Appl. Mater. Interfaces 7 (2015) 14983-14991.

[28] X.Y. Liu, Y. Guan, X.B. Ding, Y.X. Peng, X.P. Long, X.C. Wang, K. Chang, Design of temperature sensitive imprinted polymer hydrogels based on multiple-point hydrogen bonding, Macromol. Biosci. 4 (2004) 680-684.

[29] X.Y. Liu, T. Zhou, Z.W. Du, W. Zhong, J.H. Zhang, Recognition ability of temperature responsive molecularly imprinted polymer hydrogels, Soft Matter 7 (2011) 1986-1993.

[30] J.M. Zhuang, Mallory R. Gordon, J. Ventura, L.Y. Li, S. Thayumanavan, Multi-stimuli responsive macromolecules and their assemblies, Chem. Soc. Rev. 42 (2013) 7421-7435.

[31] X.J. Li, B.L. Zhang, W. Li, X.F. Lei, X.L. Fan, L. Tian, H.P. Zhang, Q.Y. Zhang, Preparation and characterization of bovine serum albumin surface-imprinted thermosensitive magnetic polymer microsphere and its application for protein recognition, Biosens. Bioelectron. 51 (2014) 261-267.

[32] L.C. Xu, J.M. Pan, J.D. Dai, X.X. Li, H. Hang, Z.J. Cao, Y.S. Yan, Preparation of thermal-responsive magnetic molecularly imprinted polymers for selective removal of antibiotics from aqueous solution, J. Hazard. Mater. 233-234 (233) (2012) 48-56.

[33] G. Moad, E. Rizzardo, S.H. Thang, Living radical polymerization by the RAFT process, Aust. J. Chem. 58 (2005) 379-410.

[34] F.F. Duan, C.Q. Chen, L. Chen, Y.J. Sun, Y.W. Wang, Y.Z. Yang, X.G. Liu, Y. Qin, Preparation and evaluation of water-compatible surface molecularly imprinted polymers for selective adsorption of bisphenol A from aqueous solution, Ind. Eng. Chem. Res. 53 (2014) 14291-14300.

[35] J.Z. Liu, W.Z. Wang, Y.F. Xie, Y.Y. Huang, Y.L. Liu, X.J. Liu, R. Zhao, G.Q. Liu, Y. Chen, A novel polychloromethylstyrene coated superparamagnetic surface molecularly imprinted core-shell nanoparticle for bisphenol A, J. Mater. Chem. 21 (2011) 9232-9238.

[36] Y.M. Ren, W.Q. Ma, J. Ma, Q. Wen, J. Wang, F.B. Zhao, Synthesis and properties of BPA molecularly imprinted particle for selective recognition of BPA from water, J. Colloid Interface Sci. 367 (2012) 355-361.

[37] N. Griffete, H. Li, A. Lamouri, C. Redeuilh, K. Chen, C.Z. Dong, S. Nowak, S. Ammar, C. Mangeney, Magnetic nanocrystals coated by molecularly imprinted polymers for the recognition of BPA, J. Mater. Chem. 22 (2012) 1807-1811.

[38] Z.K. Lin, W.J. Cheng, Y.Y. Li, Z.R. Liu, X.P. Chen, C.J. Huang, A novel superparamagnetic surface molecularly imprinted nanoparticle adopting dummy template: an efficient solid-phase extraction adsorbent for bisphenol A, Anal. Chim. Acta 720 (2012) 71-76.

[39] I. Naoko, O. Tooru, T. Takeuchi, Hydrophilic molecularly imprinted polymers for bisphenol A prepared in aqueous solution, Microchim. Acta. 180 (2013) 1387-1392.

[40] Y.Z. Wu, L.Z. Zhuang, B.B. Ma, S.X. Chen, X.Z. Xu, Y.Q. Zeng, Preparation of a surface molecularly imprinted fiber for bisphenol a recognition, J. Polym. Res. 468 (2014) 1-9.

[41] X.Q. Wu, Z. Zhang, J.H. Li, H.Y. You, Y.B. Li, L.X. Chen, Molecularly imprinted polymers-coated gold nanoclusters for fluorescent detection of bisphenol A Sens. Actuators B: Chem. 211 (2015) 507-514. 